\title{
BioSAXS-2000 AUTO: Biological solution scattering in the home laboratory
}

\author{
Angela Criswell $^{1}$, Mark Del Campo $^{1}$, Thom Hendrixson ${ }^{1}$, Colin Acheson ${ }^{1}$, Katsunari Sasaki $^{1}$, Pierre LeMagueres $^{1}$, Joseph Ferrara ${ }^{1}$ \\ ${ }^{1}$ Rigaku Oxford Diffraction, The Woodlands, United States \\ E-mail: angela.criswell@rigaku.com
}

Small angle X-ray scattering (SAXS) is a useful technique for extracting structural information from biological samples in solution. However, the X-ray scattering signal from macromolecules is challenging to measure because of low particle concentrations and high background from solvent. As a result, instrument designs for home laboratories, where X-ray intensities are lower than synchrotrons, require special scrutiny. In particular, collimation design, X-ray source and area detector contribute greatly to improved data quality for home laboratory experiments.

Rigaku Oxford Diffraction's BioSAXS-2000 system is a SAXS instrument for the home lab, which combines 2D Kratky collimation with confocal optics to achieve maximum X-ray flux on the sample without the need for data desmearing. The BioSAXS-2000 uses Rigaku's hybrid photon counting detector (HPC), the HyPix-3000. HPCs are ideal for measuring weak scattering from biological solutions because they combine ultra low noise, high dynamic range and direct detection of X-ray photons. In fact, HPCs are the detector of choice for most SAXS beamlines and modern home laboratory SAXS instruments. Results with the BioSAXS-2000 consistently show that this instrument produces high quality SAXS data in minutes.

The BioSAXS-2000 AUTO package includes automation tools to achieve high-throughput biological SAXS out of the synchrotron and into the home laboratory. The AUTO package consists of a 96-well Automatic Sample Changer (ASC) and an Automatic Analysis Pipeline (AAP) that uses the ATSAS ${ }^{[1]}$ package. The BioSAXS-2000, coupled to a Rigaku rotating anode source and a Rigaku HyPix-3000 hybrid pixel counting detector, collects high quality SAXS data in minutes and the AUTO package makes it possible to load a 96-well plate of samples and come back when all data collection and data processing have finished.

[1]Petoukhov, M.V., Franke, D., Shkumatov, A.V., Tria, G., Kikhney, A.G., Gajda, M., Gorba, C., Mertens, H.D.T., Konarev, P.V. \& Svergun, D.I. (2012). J. Appl. Cryst. 45, 342-350.

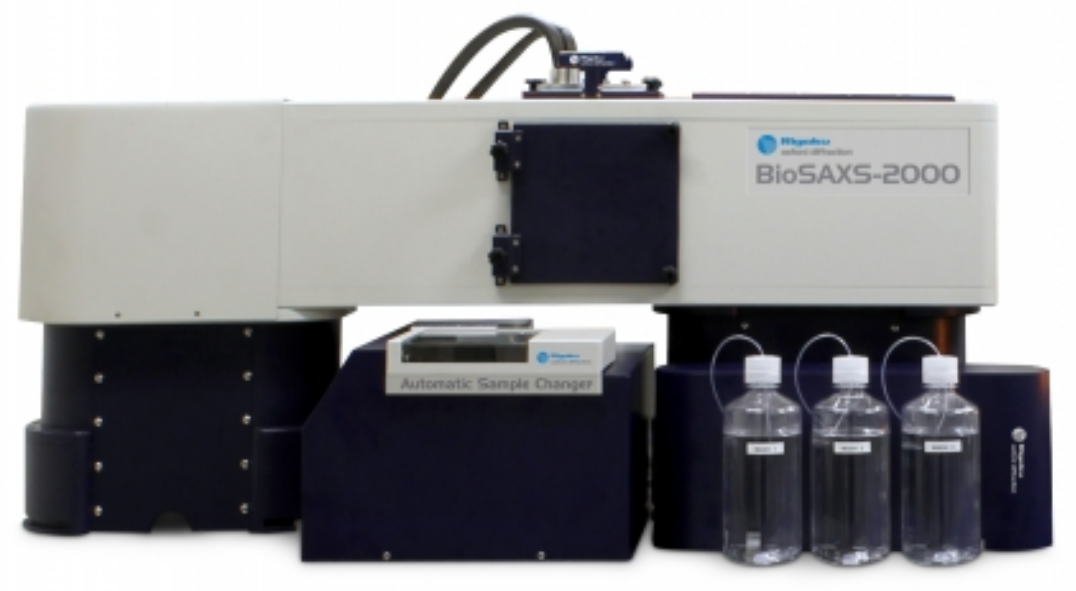

Keywords: SAXS, automation, BioSAXS, small angle scattering 